\title{
Philonsorbonne
}

\section{Merleau-Ponty et la critique des « intellectualismes »}

Lucia ANGELINO

\section{OpenEdition}

12 Journals

\section{Édition électronique}

URL : https://journals.openedition.org/philonsorbonne/144

DOI : 10.4000/philonsorbonne.144

ISSN : 2270-7336

Éditeur

Publications de la Sorbonne

\section{Édition imprimée}

Date de publication : 15 avril 2008

Pagination : 11-30

ISBN : 978-2-85944-602-4

ISSN : 1255-183X

Référence électronique

Lucia ANGELINO, « Merleau-Ponty et la critique des « intellectualismes » », Philonsorbonne [En ligne], 2 I 2008, mis en ligne le 28 janvier 2013, consulté le 08 juin 2021. URL : http://journals.openedition.org/ philonsorbonne/144; DOI : https://doi.org/10.4000/philonsorbonne.144 


\title{
Merleau-Ponty et la critique des « intellectualismes »
}

\author{
Lucia Angelino
}

La Phénoménologie de la perception est un ouvrage important et original qui amorce une nouvelle conception de la philosophie, se situant à la charnière entre la description et la spéculation. Notre texte tente d'en dégager la signification, en accordant une attention particulière à la notion de « réflexion radicale», sous laquelle cet auteur thématise et réfléchit son propre geste philosophique, et en adoptant pour fil conducteur la critique de l'intellectualisme sur laquelle l'ouvrage insiste particulièrement ${ }^{1}$.

Nous avons pris le parti d'approcher la signification philosophique du concept de «réflexion radicale » de manière négative, à partir des critiques que Merleau-Ponty adresse à ce type particulier de réflexion philosophique la réflexion intellectualiste ou cartésienne - qui a eu tendance à dominer la scène philosophique française, au moment où il commençait ses recherches sur la perception, en 1933-34. Un tel choix méthodologique s'explique facilement: étant donné que Merleau-Ponty débat sans cesse avec l'intellectualisme, tout d'abord pour critiquer cette forme particulière de la réflexion que pratiquait cette tradition de la philosophie française, il nous a semblé pertinent de déduire, à partir de ce qu'il récusait chez cet adversaire, ce qu'il se proposait de faire. Régresser en direction de la réflexion intellectualiste ou cartésienne, puis s'acheminer vers le «nouveau genre de réflexion »-dite radicale - proposée comme substitut consistera, par suite, à s'avancer dans la détermination de la philosophie vers laquelle il nous amène, au moins en intention.

Nous nous proposons ici, pour commencer, d'expliciter le sens propre de la notion de « réflexion radicale », et d'expliquer en quoi elle se distingue

1. Ce texte, qui reprend les thèmes abordés dans notre thèse de doctorat sur Merleau-Ponty et le problème de la réflexion, a été présenté dans le cadre d'une journée doctorale organisée par l'équipe EXeCO de l'Université de Paris 1/Panthéon-Sorbonne, à Paris, le 3 mars 2007. 
de la réflexion intellectualiste ou cartésienne. Deux temps rythmeront notre parcours: dans une première partie, nous examinons, en deçà de ses différentes déclinaisons, les traits caractéristiques et stables du «geste » réflexif/intellectualiste que conteste Merleau-Ponty, les invariants qui en font un style unique et commun à plusieurs auteurs enrôlés par le philosophe sous la bannière de l'intellectualisme. Dans une deuxième partie, nous allons essayer de construire, par contraste et symétrie, les traits qui font la particularité de la réflexion radicale par laquelle Merleau-Ponty projette de remplacer le modèle réflexif ou intellectualiste de la réflexion, jugé désormais dépassé et inadéquat depuis la rencontre en 1934 de la phénoménologie allemande de Husserl. Ces deux volets permettront la formulation d'un ensemble de remarques critiques sur les grandes lignes et les limites de la nouvelle conception de la philosophie que cherche à réaliser Merleau-Ponty.

\section{La critique merleau-pontienne de l'intellectualisme}

L'intellectualisme n'a jamais cessé de hanter Merleau-Ponty, si bien qu'il serait pertinent de considérer son œuvre entière comme une critique vaste et étoffée de cette longue tradition de la philosophie française - qui est une tradition cartésienne - dans laquelle elle s'insère et plonge ses racines. Cet intérêt critique et polémique se comprend facilement. Si Merleau-Ponty conteste sans cesse l'intellectualisme, c'est tout d'abord pour repousser les interprétations intellectualistes de la perception qui étaient dominantes au moment où il commençait ses recherches; bien conscient d'emprunter la problématique de la perception aux philosophies intellectualistes ou réflexives $^{2}$, il vise d'abord à se positionner par rapport aux travaux de ses prédécesseurs illustres, à ce qu'on appelle - à la suite de G. Granel "l'école française de la perception ». En outre, s'il consacre autant de temps et d'énergie à critiquer l'intellectualisme, c'est aussi, de manière plus générale, parce que, comme il l'explique en 1946 dans un entretien avec Maurice Fleuret, il essaie dans la Phénoménologie de la perception de répondre à une question qu'il partage avec les philosophes de sa génération qui est la suivante : " comment sortir de l'idéalisme sans retomber dans la naïveté du réalisme? ${ }^{4}$, c'est-à-dire comment surmonter le travers de l'intellectualisme, ainsi que les dualismes du subjectif et de l'objectif, de la pensée et de la matière qui en constituent l'impasse, sans retomber dans « la

2. Comme il le déclare lui-même dans la conférence qu'il présente à la Société Française de Philosophie en 1946 : «En avançant la thèse d'un primat de la perception, nous avons moins le sentiment de proposer une nouveauté que de mener jusqu'à leurs conséquences les travaux de nos aînés ", M. Merleau-Ponty, Le primat de la perception et ses conséquences philosophiques, Lagrasse, Verdier, 1996, p. 72.

3. G. Granel, Traditionis traditio, Paris, Gallimard, 1972, p. 19.

4. M. Merleau-Ponty, Parcours 1935-1951, Paris, Verdier, 1997, p. 66. 
croyance absolue » - à laquelle restait l'empirisme - «au monde comme totalité des événements spatio-temporels $»^{5}$, comme monde en soi, défini par l'extériorité de ses parties, où le sujet ne serait qu'un objet parmi d'autres. Si l'auteur de la Phénoménologie va si loin dans la critique de l'intellectualisme, c'est donc non seulement parce qu'il repousse les théories intellectualistes de la perception, mais encore parce qu'il cherche à réaliser une philosophie du monde vécu (ontologie), se situant entre le réalisme et l'idéalisme. Et plus particulièrement parce qu'il tente d'occuper une position médiane entre ces deux pôles extrêmes de la pensée, d'en dépasser l'antinomie en vue de comprendre «comment paradoxalement il y a pour nous de l'en soi $»^{6}$.

L'enjeu de la critique merleau-pontienne de l'intellectualisme peut être formulé ainsi : il s'agit de reprendre le problème de la perception et particulièrement de la perception du corps propre et par là, de nous remettre en présence du monde qui filtre à travers elle, le monde tel qu'il nous apparait «en tant que nous sommes au monde par notre corps, en tant que nous percevons le monde avec notre corps $»^{7}$. Bref, il s'agit de « revenir au monde vécu en deçà du monde objectif $»^{8}-$ qui n'est qu'une version du monde, le monde saisi depuis le point de vue d'un Ego transcendantal central et unique - et d'en réhabiliter la priorité sur le monde propre à la réflexion, il s'agit encore de mettre à jour la richesse cachée et inépuisable qu'il possède en lui-même. Avant d'entrer dans les grandes lignes de la polémique que l'auteur de la Phénoménologie entretient avec la tradition réflexive ou intellectualiste et afin de spécifier les divers éléments constitutifs des intellectualismes dont il sera question ici, il faut d'abord préciser le double sens dont se trouve investie cette appellation chez lui.

\section{L'intellectualisme au sens large et au sens étroit}

L'intellectualisme au sens large désigne toute doctrine philosophique issue de l'idéalisme kantien et, plus loin, cartésien, le plus couramment pratiquée par une famille de penseurs différents - comprenant non seulement Descartes, Kant, Alain et Lagneau, Lachelier, Brunschvicg, Lachièze-Rey, mais aussi une psychologie rationaliste et intellectualiste encore dépendante du schéma et de la psychologie kantiens des facultés - qui a tendance a croire que «le sujet méditant puisse absorber dans sa méditation ou saisir sans reste l'objet sur lequel il médite, notre être se ramener à notre savoir »", bref toute doctrine qui a tendance à réduire l'existence à la pensée, l'objet de la connaissance au sujet de la connaissance.

\footnotetext{
5. M. Merleau-Ponty, Phénoménologie de la perception, op. cit., p. 50.

6. Ibid., p. 86.

7. Ibid., p. 239.

8. Ibid., p. 69.

9. Ibid., p. 76
} 
L'intellectualisme au sens restreint n'est que l'adversaire apparent de l'empirisme et plus généralement du réalisme ${ }^{10}$ : la position inverse et symétrique à celle qui s'exprime surtout dans la science mécaniste physicaliste des XVII ${ }^{\mathrm{e}}$ et XVIII ${ }^{\mathrm{e}}$ siècles « selon laquelle les consciences sont insérées dans le tissu du monde objectif et des événements en soi » ${ }^{11}$ et qui engendre la perception à partir d'un «univers, c'est-à-dire d'une totalité achevée, explicite, où les rapports soient de détermination réciproque $»^{12}$. Nous voudrions ici examiner de manière spécifique la polémique que Merleau-Ponty entretient avec l'intellectualisme, entendu au sens large comme doctrine qui a tendance à réduire l'objet de la connaissance au sujet de la connaissance et qui cherche par la suite à décomposer le tissu intentionnel selon la dualité conceptuelle de la conscience et de l'objet, sans pour autant entrer dans les détails des discussions qu'il entretient singulièrement avec chacun de ces auteurs, ses différents usages et figurations historiques.

\section{Illusions, excès et simplifications de la réflexion intellectualiste}

En plusieurs passages, parfois très fouillés et minutieux, où il discute autant avec ses prédécesseurs qu'avec ses contemporains, Merleau-Ponty nous révèle quels sont les excès et les simplifications principales de l'intellectualisme qu'il cherche à surmonter. Tout d'abord il met en question la notion de conscience, suivant l'acception héritée de Kant et plus loin de Descartes, et son primat absolu, lié au fait qu'elle est «conscience de part en part ${ }^{13}$, une conscience transparente pour elle-même et dominatrice/souveraine sur le champ de son expérience, constituant l'univers devant elle et saisissant les objets mêmes dans une expérience indubitable ${ }^{14}$. En d'autres termes, il faut contester le primat absolu de la conscience cartésienne, lié au fait que, après avoir purifié simultanément les notions communes du corps et de l'âme, l'on pense qu'elle puisse se déployer sur un plan de transparence totale, devenir toute entière conscience de soi et du monde. Or, Merleau-Ponty pensait que le cogito - la pure apparition de quelque chose à quelqu'un, et d'abord de moi à moi - ne doit pas être compris à la lettre comme l'attestation d'un être dont toute l'essence est de se savoir, c'est-à-dire d'être conscience. C'est une illusion intellectualiste de

10. Même si notre examen de la lutte que Merleau-Ponty mène contre l'intellectualisme se limite à la Phénoménologie de la perception, je signale, au passage, que l'on peut en trouver facilement les prémisses et les lignes principales dans la Structure du comportement.

11. M. Merleau-Ponty, Phénoménologie de la perception, op. cit., p. 489-490.

12. Ibid., p. 85 .

13. Ibid., p. 231.

14. Elle «constitue tout» et «éternellement possède la structure intelligible de tous ses objets », ibid., p. 36 . 
croire que nous puissions devenir « tout entier conscience, nous ramener à la conscience transcendantale ${ }^{15}$ et que cette illusion est à la racine même de cette autre erreur, cet aveuglement fondamental, qui consiste à croire que nous puissions disposer ou posséder, sous notre regard, le monde entier, notre histoire et les objets perçus, déployés et objectivés comme des systèmes transparents de relation, nous élever à une vue totale sur le monde - soit-il intérieur ou extérieur. Comme il l'explicite lui-même dans le passage suivant qui nous parait particulièrement significatif : "L'erreur des philosophies réflexives est de croire que le sujet méditant puisse absorber dans sa méditation ou saisir sans reste l'objet sur lequel il médite, notre être se ramener à notre savoir. (...) Nous ne pouvons pas davantage devenir tout entier conscience, nous ramener à la conscience transcendantale », déployer une conscience invisible sur un plan de transparence totale. "Si nous étions la conscience, nous devrions avoir devant nous le monde, notre histoire, les objets perçus dans leur singularité comme des systèmes de relations transparentes $»^{16}$. En résumé, Merleau-Ponty reproche à la réflexion intellectualiste de reposer sur l'idée cartésienne d'une conscience souveraine et transparente pour elle-même, à laquelle rien ne peut être étranger - qui vaut juste autant que celle, empiriste, d'une objectivité absolue, puisque "par là, toutes les thèses de l'empirisme se trouvent » simplement « renversées » 17 et « le préjugé d'un univers en soi parfaitement explicite ${ }^{18}$, réaffirmé une deuxième fois, maladroitement travesti et dépaysé mais bien reconnaissable. En d'autres termes, la réflexion intellectualiste est critiquée tout d'abord en raison de la complicité entre la position d'une subjectivité transcendantale ou conscience constituante absolue - qui contemple ou objective le monde «d'ailleurs et de nulle part » après l'avoir construit (qui en possède par-devers soi la loi, la cléél et le « secret de fabrication ») et le soi-disant Kosmothéoros, ou démon laplacien, qui est un sujet spectateur, extérieur et ontologiquement dominateur sur le champ de son expérience. Depuis une telle posture de détachement à l'égard du perçu - que Merleau-Ponty qualifie de «surplomb» ou de survol l'intellectualisme et la pensée de la science partagent la même erreur fondamentale qui est de dénier ou de neutraliser (de faire abstraction) la perception originaire, par laquelle le monde ne se déploie que peu à peu, jamais « en entier », partiellement et imparfaitement, et son rôle initiatique.

15. Ibid., p. 76.

16. M. Merleau-Ponty, Phénoménologie de la perception, op. cit., p. 76.

17. Ibid., p. 241.

18. Ibid., p. 51.

19. Une telle critique et une telle collusion entre la pensée de la science - pensée de survol et la position du sujet comme œil, comme spectateur étranger au monde, ou comme point de vue de nulle part, ont été développées ultérieurement par Merleau-Ponty dans Le visible et l'invisible et L'œil et l'esprit. 
Merleau-Ponty va encore critiquer la réflexion intellectualiste, en tant que prise dans une naïveté encore plus grave qui est une naïveté quant à ellemême. Aveuglée par l'idée de rejoindre à l'intérieur de l'homme «un pouvoir constituant qui a toujours été lui $»^{20}$ - c'est-à-dire la prémisse, ou la condition de possibilité qui depuis toujours soutenait l'expérience, la réflexion intellectualiste "perd conscience de son commencement ${ }^{21}$ ou si l'on préfère, entretient une idée chimérique de son commencement, comme origine d'avant l'origine, ayant son siège dans une subjectivité transcendantale qui aurait préséance sur toutes les autres. Elle oublie en d'autres termes la vie de conscience irréfléchie et pré-personnelle qui la précède et d'où elle émerge. L'oubli de la vie de conscience irréfléchie ou perceptive qui habite comme un «cimetière marin ${ }^{22}$ le sujet conscient et réflexif qui ne fait qu'un avec le mouvement de l'existence corporelle, explique aussi que l'intellectualisme utilise tacitement ce que Merleau-Ponty appelle la «foi primordiale» ou la «croyance au monde» ou «l'opinion originaire », c'est-à-dire ce savoir de position et de situation antéprédicatif et pré-objectif du monde qui m'est donné avec ce premier ici ou visée primordiale, qui est le corps (ou si l'on préfère avec notre condition corporelle comme système des conditions anonymes - la vie de conscience pré-personnelle et donnée - sur le fond de laquelle s'établit «tout acte de réflexion et toute prise de position volontaire $»^{23}$ ) ; en d'autres termes une perception antérieure du monde qui m'apparaît toujours déjà faite, au moment où j'y porte attention et que je commence à essayer de la comprendre.

Oubliant la donation phénoménale primordiale du monde par la perception - « le monde qui est donné au sujet parce que le sujet est donné à lui-même $»^{24}$, le $«$ il y a du monde $»^{25}$, ou son apparaître, la réflexion traite par la suite notre expérience du monde comme l'acte d'une conscience constituante, et assimile l'acte de la perception «aux synthèses qui sont de l'ordre du jugement, des actes ou de la prédication ${ }^{26}$, remplace $«$ par une pensée éternelle "l'art caché" qui fait surgir un sens dans les "profondeurs de la nature" $\gg{ }^{27}$. L'effet en retour de cet oubli est de croire que le cogito puisse par sa pensée «égaler la richesse concrète du monde et résorber la facticité ${ }^{28}$, puisque le monde qui existe en face de lui et qu'il contemple, n'est qu'un cogitatum tout pur, que le résultat de son travail constitutif, le

20. M. Merleau-Ponty, Phénoménologie de la perception, op. cit., p. IV.

21. Ibid., p. IV.

22. Nous reprenons ici le titre de l'œuvre de P. Valéry, Cimetière marin cité par MerleauPonty dans Le visible et l'invisible, p. 91.

23. M. Merleau-Ponty, Phénoménologie de la perception, op. cit., p. 241.

24. Ibid., p. IV.

25. Ibid., p. XII.

26. Ibid., p. IV.

27. Ibid., p. 48

28. Ibid., note 1, p. 431. 
monde qu'il a constitué. L'oubli de ce monde plus originaire que nous livre la perception adéquatement analysée explique ainsi que la réflexion s'apparaisse comme une véritable création, comme possible par principe et comme tout puissante, seule à constituer le monde. Mais il s'agit, selon Merleau-Ponty, d'une naïveté, d'une réflexion incomplète, qui a perdu conscience de son commencement: "J'ai commencé de réfléchir, ma réflexion est réflexion sur un irréfléchi, elle ne peut pas s'ignorer elle-même comme événement, dès lors elle s'apparaît comme une véritable création, comme un changement de structure de la conscience, et il lui appartient de reconnaître en deçà de ses propres opérations le monde qui est donné au sujet parce que le sujet est donné à lui-même ${ }^{29}$.

La réflexion intellectualiste et la réflexion radicale divergent, non seulement quant à leur conceptualisation de la conscience, mais encore quant à leur explication de la perception, qui en est le mode d'être fondamental/originaire. Selon les théories intellectualistes classiques, à la manière de Descartes, la perception se laisse analyser et décomposer en deux éléments : ce qui est proprement vu, des données inertes, un ensemble de caractères insignifiants dont je ferai l'inventaire - par exemple quand, de ma fenêtre, je regarde des hommes qui marchent dans la rue, des mannequins vêtus, «des chapeaux et des manteaux qui peuvent couvrir des spectres et des hommes feints », dit Descartes - et, d'autre part, l'opération active d'interprétation et d'organisation (l'inspection de l'esprit, l'acte de la synthèse intellectuelle, le jugement) de ces données, produisant une perception qui leur donne une signification vivante, qui les comprend comme signifiant des hommes. Depuis la perspective de Merleau-Ponty, cette distinction, qui suppose la séparation de l'âme et du corps et l'oubli du rôle joué par le corps dans l'expérience, conduit à neutraliser l'opérativité et l'autonomie de la perception, et revient à ne plus voir le phénomène de la perception tel qu'en lui-même, c'est-à-dire un acte, et singulièrement «cet acte qui crée d'un seul coup, avec la constellation des données, le sens qui les relie, - qui non seulement découvre le sens qu'elles ont mais encore fait $q u^{\prime}$ 'elles aient un sens ${ }^{30}$. En d'autres termes, la réflexion intellectualiste voile le phénomène perceptif par le jugement qu'elle introduit comme ce qui manque au sentir pour rendre possible le percevoir.

La réflexion intellectualiste et la réflexion radicale divergent, non seulement quant à leur contenu conceptuel, mais encore quant à leur méthode. L'intellectualisme pratique une méthode ou analyse réflexive qui retrouve dans tout ce qui existe l'activité spirituelle qui l'a engendré ; c'est ainsi que par exemple la réflexion intellectualiste découvre l'œuvre de l'esprit dans la perception extérieure, «traite l'expérience du monde comme un acte de la conscience constituante ${ }^{31}$, comme le résultat du travail

29. Ibid., p. IV.

30. M. Merleau-Ponty, Phénoménologie de la perception, op. cit., p. 46.

31. Ibid., p. 281. 
constitutif de la conscience ou comme le développement d'une raison consciente universelle, en réduisant ainsi au rationnel tout ce qu'elle approche. Merleau-Ponty y voit donc une vaste pétition de principe, une vicieuse circularité, dont il tente d'amoindrir, voire d'éradiquer, la prégnance. Il considère en effet que la réflexion intellectualiste s'achève aussitôt qu'elle commence, faisant retour à une raison universelle qui est la même en tous, qui est le principe unique de toutes nos pensées - l'Ego transcendantal central et unique - et préjuge de ce qu'elle trouve par ce qu' exige cette raison universelle; par la suite elle nous oblige à investir $a$ priori le monde des conditions sans lesquelles il ne serait pas pensé, juge «de ce qui est par ce qui doit être, par ce qu'exige l'idée du savoir ${ }^{32}$. En d'autres termes, Merleau-Ponty pensait probablement que la philosophie s'abolit ou se termine, à ce point zéro (qui coïncide avec l'ego ou la « subjectivité transcendantale autonome située partout et nulle part ») où elle «ne laisserait plus rien d'implicite ou de sous-entendu dans mon savoir », «me ferait prendre possession entière de mon expérience et réaliserait l'adéquation du réfléchissant au réfléchi ${ }^{33}$, parce qu'en effet à ce point zéro (ou de nulle part), elle n' aurait plus rien à trouver ou à chercher. En outre, et ce n'est pas un moindre défaut, du seul fait qu'elle subordonne toute l'expérience «à cette conscience absolue qui le situerait dans l'ensemble de la vérité ${ }^{34}$, la réflexion intellectualiste, rend incompréhensible la variété, nivelle toutes les expériences en un seul monde, toutes les modalités de l'existence en une seule conscience et manque ainsi la consistance et la variété de notre rapport antéprédicatif et pré-objectif au monde.

Ces deux modèles de la réflexion divergent enfin par leur vocabulaire. L'auteur de la Phénoménologie critique le vocabulaire véhiculant la conceptualité philosophique de l'intellectualisme, qui repose tout entier sur le clivage et la distinction entre la conscience et l'objet qui lui est extérieur, en tant qu'inadéquat à exprimer ce que la perception et la sensation adéquatement analysées avaient mis à jour, c'est-à-dire une unité ou une connivence originaire ${ }^{35}$ entre le sujet et le monde - à la lettre une «conaissance » - irréductible à la dualité conceptuelle de la conscience et de l'objet, au vecteur intentionnel de la connaissance intellectuelle.

\section{Ibid., p. 74.}

33. M. Merleau-Ponty, Phénoménologie de la perception, op. cit., p. 73.

34. Ibid., p. 338.

35. «Moi qui contemple le bleu du ciel »- décrit-il - «je ne suis pas en face de lui un sujet acosmique, je ne le possède pas en pensée, je ne déploie pas devant lui une idée du bleu qui m'en donnerait le secret, je m'abandonne à lui, je m'enfonce dans ce mystère, il "se pense en moi", je suis le ciel même qui se ressemble, se recueille et se met à exister pour soi, ma conscience est engorgée par ce bleu illimité... », Phénoménologie de la perception, op. cit., p. 248. - «Le sujet de la sensation » - remarque-t-il - «n'est ni un penseur qui note une qualité, ni un milieu inerte qui serait affecté ou modifié par elle, il est une puissance qui conaît à un certain milieu d'existence ou se synchronise avec lui. (...) et la sensation est à la lettre une communion », Phénoménologie de la perception, op. cit., p. 245-246, les italiques sont rajoutées par nous. 
En résumé, Merleau-Ponty reproche à l'intellectualisme de thématiser notre relation primordiale au monde d'après une illégitime dualité conceptuelle - celle de la conscience et de l'objet, au lieu de demander à l'expérience elle-même son propre sens. Pour l'essentiel, l'erreur fondamentale de la réflexion intellectualiste critiquée ici tient à ce qu'elle obéit à une logique du concept, codée et figée, qui trouve en elle ses propres ressources et s'alimente elle-même, à vide, au lieu de demander à l'expérience elle-même son propre sens. Au lieu de lire et traduire la logique perceptive, inscrite directement dans le monde sensible et articulée par un système analogique d'équivalences et résonances - ce que Merleau-Ponty appelle, à la suite de Husserl, le "Logos du monde esthétique" »" du monde - la réflexion intellectualiste se contente de formuler des concepts, et dans son repli sur des tâches spéculatives, reste naïvement prise dans l'illusion et «l'auto vertige» «de pouvoir faire comprendre le monde et la vie par un agencement de concepts ${ }^{37}$.

\section{Première esquisse de la réflexion radicale obtenue par renversement du schéma intellectualiste de la réflexion}

À travers les critiques qu'il adresse à l'intellectualisme, nous commençons à apercevoir par contraste le «nouveau genre de réflexion », dite radicale que Merleau-Ponty cherche lui-même à réaliser et qui apparaît sous un certain nombre d'angles, inversée par rapport à la réflexion que pratiquait l'intellectualisme. J'essaierai donc maintenant de déduire les traits qui font la particularité de la réflexion radicale, par renversement du schéma intellectualiste, d'esquisser positivement les contours de cette notion de «réflexion radicale », par symétrie contrastée, c'est-à-dire tels qu'ils émergent au négatif des diverses lignes critiques que nous venons d'analyser.

En réaction contre une réflexion oublieuse de la vie de conscience irréfléchie et perceptive où elle s'enracine, Merleau-Ponty nous propose une réflexion se tournant vers la vie irréfléchie et encore impersonnelle, qui borde marginalement «tout acte de réflexion et prise de position volontaire ${ }^{38}$ de l'homme : la vie «de mes yeux, de mes mains, de mes oreilles qui sont autant de Moi naturels » et qui « fuse à travers moi sans que $\mathrm{j}$ 'en sois l'auteur ${ }^{39}$. Cela signifie qu' au lieu de nous installer dans un cogito inattaquable, elle part à la reconquête de la vie irréfléchie et encore impersonnelle de la corporéité, sur laquelle repose le célèbre cogito cartésien et qui poursuit à son insu dans tous les actes les plus subtils et raisonnables

36. Ibid., p. 490.

37. M. Merleau-Ponty, Sens et non-sens (1948) nouvelle éd., Paris, Gallimard, 1996, p. 35.

38. Ibid., p. 241.

39. Ibid., p. 250. 
de la conscience. Comme Merleau-Ponty le remarque à plusieurs reprises, «la réflexion n'est vraiment réflexion que si elle ne s'emporte pas hors d'elle-même, se connaît comme réflexion-sur-un-irréfléchi, et par conséquent comme un changement de structure de notre existence ${ }^{40}$.

Cette remarque fondamentale permet en outre à Merleau-Ponty de mettre en suspens toute tentative ou ambition philosophique/réflexive de maîtrise et de «survol» sur le monde. En effet, l'enracinement du cogito dans un être pré-réflexif incarné, qui voit le monde d'ici, aujourd'hui, est absolument incompatible avec l'attitude de désengagement et détachement caractéristiques de ce que Merleau-Ponty appelle la position de surplomb du spectateur ou Kosmothéoros - posture (et délire) définitoire(s) du cogito intellectualiste tenant le monde devant soi à titre d'objet à inspecter. (« Le cogito intellectualiste »-remarque Merleau-Ponty - ne laisse en face de lui qu'un cogitatum tout pur qu'il possède et constitue de part en part ${ }^{41}$.

Combattre cette forme d'intellectualisme consiste donc à revenir sur le contact naïf du corps avec le monde, sur le véritable rôle initiatique de la perception et sur le lien pré-réflexif de l'esprit et du monde. Il s'ensuit qu'en réaction contre la méthode réflexive qui décolle de l'expérience, et s'aliène cette dernière, pour remonter au sujet comme à une condition de possibilité distincte d'elle et du point de vue duquel notre expérience irréfléchie ou corporelle du monde n'est qu'un point de départ naïf à congédier et la matière sensible n'est qu'une pure diversité ou multiplicité (le divers sensible ou empirique) en soi, informe et désarticulée, donnée au titre de matière inerte à son acte transcendantal (la synthèse intellectuelle ou l'inspection de son esprit, le jugement), Merleau-Ponty nous propose une réflexion qui découvre derrière elle, comme sa propre condition de possibilité, une expérience plus fondamentale - l'expérience pré-réflexive des synthèses perceptives/passives inscrites dans le corps - où les données qu'elle prétend éclairer se trouvent déjà présentes, sans l'être encore à titre d'objets. Cela signifie qu'elle n'a plus à découvrir, dans un Ego transcendantal, ou une conscience constituante et législatrice, la signification qu'elle avait donnée au monde, ni à dégager les conditions générales qui rendent possible un monde pour un tel sujet. À l'inverse, la tâche d'une réflexion radicale consiste, d'une manière paradoxale, à faire retour à notre expérience irréfléchie du monde pour en expliciter les formations et «pour replacer en elle l'attitude de vérification et les opérations réflexives, et pour faire apparaitre la réflexion comme une des possibilités de mon être ${ }^{42}$, comme une activité émergeant d'une dimension de passivité plus fondamentale : mon enracinement dans le monde. Bien évidemment une telle réflexion, ni ne trouve en elle ses propres ressources, ni ne se réalise d'avance dans l'irréfléchi; au contraire, «nous devons la considérer

40. Ibid., p. 76.

41. Ibid., p. 387-388.

42. Ibid., p. 279. 
comme une opération créatrice qui participe elle-même à la facticité de l'irréfléchi $\gg{ }^{43}$.

Une philosophie comme réflexion radicale n'a donc plus son centre dans « une subjectivité transcendantale autonome, située partout et nulle part ${ }^{44}$, qui contemple le monde «d'ailleurs et de nulle part » après l'avoir construit, c'est-à-dire dans ce point zéro qui « me ferait prendre possession entière de mon expérience et réaliserait l'adéquation du réfléchissant au réfléchi ${ }^{45}$. Elle se situe là même où elle commence, c'est-à-dire au cœur d'une expérience phénoménale, où ne règne plus en totale maîtrise un Ego transcendantal central et démiurge universel, qui en possèderait par avance la structure et la loi, mais un sujet incarné, privé de son pouvoir souverain et de son statut privilégié (aussi bien par rapport aux autres sujets que par rapport au monde), qui commence à réfléchir sur ce quelque chose dont il a eu la perception.

Dès lors, la finalité de la réflexion se trouve ainsi redéfinie. Il s'agit désormais et conformément à la méthode inaugurée par Husserl, «de décrire, et non pas d'expliquer ni d'analyser ${ }^{46}$, de tenter «l'essai d'une description directe de notre expérience telle qu'elle est ${ }^{47}$, bref d'étudier ce qui apparaît, ce dont nous pouvons vraiment avoir l'expérience, par opposition à ce qui ne serait que construction des concepts, en d'autres termes «l'apparition de l'être à la conscience »" ${ }^{48}$ le phénomène. La méthode descriptive, inaugurée par Husserl et pratiquée par la psychologie, est ici le mode d'approche approprié, afin de respecter la nature des phénomènes. Cela ne signifie pourtant pas que Merleau-Ponty caresse l'ambition de laisser le phénomène indemne, qu'il pense que la philosophie puisse « laisser les choses en l'état». Au contraire, conscient de l'ambiguïté fondamentale qui peut être liée au geste réflexif, Merleau-Ponty dédouble la tâche de la réflexion. Comme geste ambigu - à la fois fidèle et méfiant - qui ne peut prétendre laisser intact son objet, la réflexion n'est radicale que si elle nous rend «compte de la transformation qu'elle entraîne avec elle dans le spectacle du monde et dans notre existence ${ }^{49}$. Cela signifie qu'il s'agit de réfléchir sur une expérience irréfléchie, en incluant le fait qu'une telle réflexion modifie la représentation spontanée de celle-ci et a tendance à se dissimuler à elle-même. C'est pourquoi une réflexion radicale est «non seulement opérante, mais encore consciente d'elle-même dans son opération $»^{50}$ : elle est donc une réflexion autocritique.

43. Ibid., p. 74.

44. Ibid., p. 75.

45. Ibid., p. 73.

46. Ibid., p. II.

47. Ibid., p. I.

48. Ibid., p. 74.

49. Ibid., p. 75.

50. Ibid., p. 253. 
L'on pourrait dire que la réflexion radicale se laisse en outre caractériser - par contraste avec la réflexion intellectualiste - comme tentative de «faire parler» notre premier contact naïf avec le monde, qui s'établit par la perception sensible. Conformément à la tâche que Husserl fixait à la phénoménologie, c'est cette expérience irréfléchie et muette encore, qu'il s'agit d'amener à exprimer son sens. Dès qu'il s'agit de traduire sans trahir l'expérience perceptive qui est - dit-il - «la communication d'un sujet fini avec un être opaque d'où il émerge mais où il reste engagé ${ }^{51}$, «la reprise ou l'achèvement par nous d'une intention étrangère ou inversement l'accomplissement au-dehors de nos puissances perceptives et comme un accouplement de notre corps avec les choses ${ }^{52}$ donc une expérience irréductible (impensable à partir de) à la dualité conceptuelle de la conscience et de l'objet, le discours et le vocabulaire réflexif se trouvent inévitablement mis en suspens et redéfinis. Réfléchir, c'est non pas «thématiser» - l'objet et la conscience - et les conduire au concept, mais à l'inverse « retrouver en deçà de l'idée du sujet et de l'idée de l'objet, le fait de ma subjectivité et l'objet à l'état naissant, la couche primordiale où naissent les idées comme les choses $»^{53}$. D'où le travail épuisant et interminable d'une réflexion radicale, qui doit me ressaisir « pendant que je suis en train de former et de formuler l'idée du sujet et celle de l'objet», mettre «au jour la source de ces deux idées», être une « réflexion non seulement opérante, mais encore consciente d'elle-même dans son opération ${ }^{54}$. Dire que la réflexion doit être non seulement opérante, mais encore consciente d'elle-même dans son opération, c'est dire qu'elle doit prendre du recul par rapport à elle-même et par rapport à son discours et au langage qu'elle utilise et mobilise, survoler ce qu'elle exprime, et se survoler elle-même dans ce qu'elle exprime. Manifestement, une réelle difficulté compromet ou fragilise un tel retour de la réflexion sur elle-même, cette tentative de la réflexion de faire retour sur elle-même, pour se comprendre dans l'exercice de son activité de connaissance, de même que pour mettre en scène et donner à voir la manière dont elle comprend son propre travail et ses opérations. C'est comme si l'on demandait à l'œil de se voir lui-même. Manifestement ce genre d'opération pourrait se poursuivre à l'infini comme dans le dessin d'Escher, sans que la réflexion puisse jamais parvenir à une vue entièrement déployée d'elle-même. Il est curieux de constater au passage que la réflexion radicale débouche sur un paradoxe, peut-être un véritable problème, qui est plus souvent traité par les « logicistes » par ailleurs si éloignés d'une philosophie telle que celle qui nous occupe, traitant du langage : on n'arrive jamais à percer le mur du langage, parce que c'est encore en lui que l'on se situe encore pour discourir sur lui et en établir la validité ou les lois. Il s'agit toutefois d'un problème

51. Ibid.

52. Ibid., p. 370.

53. Ibid., p. 254.

54. Ibid., p. 253. 
que l'auteur de la Phénoménologie de la perception ne parvient pas à maîtriser (et ne prétend d'ailleurs pas maîtriser), tant s'avèrent grandes les puissances qui entrent en lice. Il s'agit peut-être davantage d'un problème qui permet de deviner, sans justement pouvoir les préciser ou les définir rigoureusement, les limites de toute recherche, d'un problème qu'on doit espérer résoudre définitivement et intégralement.

Reste à souligner qu'à partir de là, nous pourrions cependant mesurer tout le chemin parcouru depuis la Phénoménologie de la perception.

Récapitulons à présent les conclusions que nous pouvons tirer de cette étude. Faisant fond sur le procédé des contrastes, nous avons découvert finalement :

a/ ce qu'est la réflexion pour Merleau-Ponty: une activité émergeant d'une dimension de passivité plus fondamentale, d'une vie de conscience irréfléchie, pré-personnelle qui ne fait qu'un avec le mouvement de l'existence corporelle: quoique libre, la réflexion ne pourrait surgir si la conscience humaine ne se prêtait pas à cet avènement d'une ouverture/initiation sensible au monde et à soi, antérieure à la réflexion thétique et explicite d'un sujet qui revient sur soi et ses actes et éminemment liée au corps et à la perception.

b/ ce qu'elle doit faire : revenir sur le contact naïf (ou le «pacte naturel ») reliant le corps au monde, sur le rôle initiatique de la perception et sur le lien pré-réflexif de l'esprit et du monde, partir à la reconquête de la vie irréfléchie et encore impersonnelle de la corporéité, qui préfigure et annonce l'acte réflexif et qui se poursuit dans tous les actes plus subtils et raisonnables de la conscience.

c/ et comment elle doit le faire : d'une part, en renonçant au vocabulaire $\mathrm{du}$ dualisme et au point de vue de la conscience objectivante, dans une tentative risquée de refondre la conceptualité philosophique en vue d'exprimer et de respecter l'unité ou la connivence originelle du moi et du monde, de l'intérieur et de l'extérieur. Et d'autre part, cela s'accomplit par une destitution de l'idéal et de la posture métaphysique générale du Kosmothéoros, c'est-à-dire du «voyant qui oublie qu'il a un corps [...], qui se fait visionnaire ${ }^{55}$, du sujet spectateur, contemplatif et extérieur, ontologiquement dominateur sur le champ de son expérience.

Plusieurs questions critiques se posent à l'approche du «nouveau genre de réflexion » proposé par Merleau-Ponty, en particulier celle de savoir s'il nous amène à une réforme sans dépassement ou à une véritable sortie de l'intellectualisme. On semble s'accorder sur le fait que dans la Phénoménologie de la perception l'intellectualisme est, comme l'exprime bien V. Descombes, «réformé, mais non surmonté » ${ }^{56}$. Certes, le renversement du schéma intellectualiste de la réflexion, opéré par MerleauPonty, ne change rien quant aux structures de base de cette philosophie : la

55. M. Merleau-Ponty, Le visible et l'invisible, Paris, Gallimard, 1964, p. 108.

56. V. Descombes, Le même et l'autre, Paris, Les éditions de Minuit, 1979, p. 88. 
conscience $^{57}$ et la corrélation noético-noématique. Si loin qu'il aille dans l'exploration d'une vie de conscience irréfléchie, à partir de laquelle l'homme conscient doit se ressembler lui-même, Merleau-Ponty respecte/accepte pourtant l'essentiel de ce qu'enseigne la philosophie depuis Descartes : c'est toujours l'idée d'un face à face entre la conscience et l'objet, que l'on retrouve jusque dans les analyses du corps et de son dialogue avec les choses. Sans vouloir nous engager plus loin dans cette controverse, nous voudrions signaler que l'analytique du corps propre, développée dans la première partie de la Phénoménologie de la perception, donne l'amorce à une voie de sortie de l'intellectualisme plus viable et prometteuse - quoique encore discrète et implicite - que celle explicitement indiquée dans le chapitre sur le Cogito.

\section{IV. À quelle philosophie conduirait une réflexion-sur-l'irréfléchi ?}

Avant de conclure, il reste à se demander ce que devient la philosophie envisagée comme réflexion radicale : une fois critiquée la réflexion que pratiquait l'intellectualisme, ne serons-nous pas réduits à une conception de la philosophie comme monstration immédiate de la réalité, évocation, plus proche d'une fascination ou d'une suggestion, telles que peut nous les peut procurer un simple récit ou une œuvre d'art ?

Il est intéressant de noter qu'à son origine même, la tentative entreprise par Merleau-Ponty de réflechir-sur-l'irréfléchi, en tant que tentative de sortir de la philosophie traditionnelle - issue de l'idéalisme kantien et plus loin cartésien (et plus loin encore platonicien) et de dépasser la tradition rationaliste en général, a été envisagée et reçue avec suspicion et méfiance. On se rappelle à cette égard, qu'en 1946, lors d'une séance de la Société française de Philosophie où Merleau-Ponty présentait et défendait les principales thèses et idées exposées dans la Phénoménologie de la perception, il avait à se défendre contre l'accusation, récurrente, d'être « allé trop loin » dans la critique de l'intellectualisme et d'avoir «inverti le sens ordinaire de ce que nous appelons $»$ depuis Platon $«$ la philosophie $»^{58}$. Tout particulièrement, É. Bréhier - qui était parmi les participants à cette séance considère que chez Merleau-Ponty la philosophie revêt un sens nouveau et tout à fait déconcertant. À ses yeux, la démarche entreprise par le jeune philosophe soulève en effet la question de savoir «si la philosophie consiste à s'engager dans le monde, à s'engager dans les choses, non pas au point de

\footnotetext{
57. Comme le montre très clairement R. Barbaras, le concept de conscience incarnée et perceptive est un concept instable qui se critique en quelque sorte lui-même. Cf. R. Barbaras, «Conscience et perception. Le cogito dans la Phénoménologie de la perception», Le tournant de l'expérience, Paris, Vrin, 1998, p. 159-183.
}

58. M. Merleau-Ponty, Le primat de la perception et ses conséquences philosophiques, op. cit., p. 73. 
s'identifier à elles, mais au point de suivre toutes leurs inflexions, ou bien si la philosophie ne consiste pas dans une marche précisément inverse de cet engagement ${ }^{59}$. Pour Bréhier - qui s'inscrit dans la tradition philosophique issue de Platon - la philosophie suppose toujours et nécessairement un détachement à l'égard du perçu en tant que région confuse des apparences sensibles : penser c'est prendre ses distances vis-à-vis de la perception et s'élever par le progrès de la connaissance à une représentation du monde intelligible, qui soit cohérente, qui satisfasse la raison et qui suppose une autre faculté de connaître que la perception.

De son point de vue, le dépassement de la tradition philosophique proposé par Merleau-Ponty lui paraît impliquer une retombée de la philosophie dans le métaphorique ou le poétique. Bréhier s'exprime ainsi avec force : «je vois vos idées s'exprimant par le roman, par la peinture, plutôt que par la philosophie. Votre philosophie aboutit au roman. Ce n'est pas un défaut, mais je crois vraiment qu'elle aboutit à cette suggestion immédiate des réalités telle qu'on la voit dans les œuvres des romanciers ${ }^{60}$.

Pour sa part, Merleau-Ponty répond de «biais » à cette remarque de taille. Cela suggère qu'à cette époque le jeune philosophe n'assume, ni ne domine totalement les conséquences métaphilosophiques de ses options philosophiques, auxquels Bréhier l'oblige à penser. Il est encore loin de posséder tous les instruments conceptuels qui lui seraient nécessaires pour entretenir une discussion sérieuse avec cette position.

Cette remarque critique est, à notre avis, l'une des plus pertinentes qui ont été adressées à Merleau-Ponty à son époque et nous aide à mieux comprendre les implications métaphilosophiques inhérentes à la démarche mise en œuvre par l'auteur de la Phénoménologie de la perception. Nous voudrions donc y revenir ici pour en exploiter tout le potentiel. Nous avons au moins deux bonnes raisons pour cela. La première tient au fait que la remarque de Bréhier situe d'emblée Merleau-Ponty au cœur d'un débat qui va opposer en 1940-45 la philosophie phénoménologique, dont tout l'effort est de revenir au monde vécu en deçà du monde objectif et de retrouver notre contact naïf avec le monde, d'une part et, d'autre part, l'épistémologie ou la philosophie intellectualiste, largement dominante à l'époque, qui au contraire ne cherchait pas à explorer ce monde concret qui reste en marge de la science puisque pour elle le seul être pensable restait défini par les méthodes de la science ${ }^{61}$. Il nous semble en effet raisonnable de croire que

59. Ibid., p. 75.

60. Ibid., p. 78.

61. Comme l'on sait pour Merleau-Ponty, c'est Léon Brunschvicg (mais également Lachelier, cf. Phénoménologie de la perception, op. cit., p. 67) et sa philosophie réflexive, qui, à son époque (début du $\mathrm{XX}^{\mathrm{e}}$ siècle) est le représentant français le plus significatif de cette philosophie, qui somme toute «se contentait de rechercher les conditions qui rendent possible » (ibid.) la pleine objectivité, l'être défini «par les méthodes de la science » (ibid.) et ne cherche pas «à explorer ce monde concret»- le monde vécu ou perçu - «qui reste en marge de la science » (Parcours II, Lagrasse, Verdier, 2000, p. 66) ou de l'univers pleinement déterminé de la science. Merleau-Ponty a témoigné de ce que sa génération devait à 
les remarques de Bréhier sur l'idée de la philosophie chez le jeune MerleauPonty expriment un conflit caractéristique de la philosophie française de l'époque entre le champ de la phénoménologie et celui de l'épistémologie suivant laquelle c'est à la science ou à une philosophie de type rationaliste de nous dire ce qui est, tout le reste étant poésie, c'est-à-dire expression subjective, savoir relatif, particularisé.

La deuxième raison pour laquelle nous sommes intéressés au débat que Bréhier entretient avec le jeune Merleau-Ponty tient à la justesse et à la perspicacité de la remarque qu'il a formulée : elle s'avère frappante, surtout si l'on considère que dans L'œil et l'esprit, dernier écrit publié du vivant de l'auteur, Merleau-Ponty nous propose une nouvelle idée de la philosophie, modelée sur l'art - et tout particulièrement la peinture moderne - comme il se trouve exprimé avec force dans le passage suivant: "Or, cette philosophie qui est à faire, c'est elle qui anime le peintre, non pas quand il exprime des opinions sur le monde, mais à l'instant où sa vision se fait geste, quand, dira Cézanne, il "pense en peinture" ${ }^{62}$. S'il est donc permis de trouver dans la remarque formulée par É. Bréhier l'intuition d'une inclination profonde qui traverse la totalité de l'œuvre de Merleau-Ponty, c'est d'elle que nous voudrions partir pour saisir l'idée, le sens profond, l'intention fondamentale qui anime et structure sa philosophie.

Comme l'on sait, il y avait aux yeux mêmes de Merleau-Ponty un lien, une proximité, une parenté profonde, entre l'activité à laquelle, en tant que philosophe, il avait consacré sa vie et l'activité à laquelle les peintres consacrent la leur. Ce lien a été discuté de manière très diverse et approfondie par des nombreux ouvrages et articles écrits et publiés lors des

l'enseignement de Léon Brunschvicg. En tant que professeur à la Sorbonne de 1909 à 1940, il exerça une autorité intellectuelle remarquable en France (vis-à-vis de Bergson, dont l'influence était au second plan et qui, en tant que professeur au Collège de France, n'avait jamais fait partie de l'Université), qui contribua à l'installation du néo-kantisme français comme doctrine dominante. Cf. La philosophie de l'existence, recueilli dans Parcours II, op. cit., p. 247 et suiv. La philosophie de Léon Brunschvicg est «la simple connaissance de l'activité spirituelle à l'œuvre dans la science » (ibid., p. 66), une réflexion sur la science, qui se contente, en d'autres termes, de rechercher les conditions de possibilité de l'objectivité scientifique, de l'univers exact de la science newtonienne. Cela ferait de Brunschvicg le représentant le plus significatif (l'incarnation) de l'intellectualisme : pour lui, « de ce qui n'a pas été réduit en objet de science, nous ne pouvons rien dire, ni penser, pas même qu'il existe » (ibid., p. 32), notre accès au monde se fait donc par la pensée, et par la pensée scientifique en particulier. C'est bien un monde partiel que me livre cette pensée, un monde, qui ne saurait être coextensif à ce qui existe. Cependant, même si l'on accordera que l'objectivité ne peut pas épuiser l'existence, «il n'y avait rien à dire de l'objet hors de ce qu'en dit la science, l'objet naturel restait pour nous une unité idéale » (Phénoménologie de la perception, op. cit., p. 67). «L'enseignement de Brunschvicg»- nous dit Merleau-Ponty «nous a appris une fois pour toutes que la science, construction de l'intelligence aux prises avec le concret, reste ouverte et ne saurait être interprétée dans un sens dogmatique. Mais la philosophie propre de Brunschvicg ne cherchait pas à explorer ce monde concret qui reste en marge de la science. La perception, l'art, la religion n'étaient selon lui que des ébauches de la connaissance scientifique », Parcours II, op. cit., p. 66.

62. M. Merleau-Ponty, L'œil et l'esprit, Paris, Gallimard, 1964. 
vingt dernières années. Curieusement, mis à part M. Carbone ${ }^{63}$, peu de commentateurs ont cherché ce qui prépare cette orientation dans les premiers textes. C'est pourquoi nous voudrions tenter ici de nous mettre en quelque sorte à la place de Bréhier pour essayer de voir ce qui, à la lecture de la Phénoménologie de la perception, a pu susciter d'abord sa réaction contre l'idée de la philosophie chez Merleau-Ponty et ensuite aussi son intuition profonde concernant sa destination et son avenir. Pour commencer, il nous semble raisonnable de croire que la réaction de Bréhier est largement motivée par la décision inaugurale qui lance le programme philosophique de la Phénoménologie de la perception et que Merleau-Ponty énonce de la manière suivante : «le premier acte philosophique serait donc de revenir au monde vécu en deçà du monde objectif, puisque c'est en lui que nous pourrons comprendre le droit comme les limites du monde objectif, de rendre à la chose sa physionomie concrète, aux organismes leur manière propre de traiter le monde, à la subjectivité son inhérence historique (...), de réveiller la perception et de déjouer la ruse par laquelle elle se laisse oublier comme fait et comme perception au profit de l'objet qu'elle nous livre et de la tradition rationnelle qu'elle fonde ${ }^{64}$. Or, voilà bien ce que MerleauPonty, du début à la fin de son œuvre n'a cessé de réclamer : au lieu de se détourner du perçu, lui accorder la primauté, s'y engager, l'habiter, l'ausculter, l'interroger, y revenir toujours. C'est contre un tel geste, à notre sens, que Bréhier réagit, puisqu'il continue de croire avec Platon que la philosophie réclame un détachement à l'égard du perçu en tant que région confuse des apparences et suppose l'exercice d'une autre faculté de connaître que la perception. On ajoutera que la réaction de Bréhier est largement motivée par l'idée de la phénoménologie explicitement thématisée dans certains passages de l'avant-propos de la Phénoménologie de la perception et même impliquée dans la démarche mise en œuvre: elle pourrait tenir à ce que Merleau-Ponty nous invite en bonne méthode phénoménologique à ne plus séparer l'ordre clair et sage des idées intelligibles de la région confuse des perceptions et apparences sensibles, à ne plus faire la différence entre d'un coté la réalité telle qu'elle est en soi et pour soi et de l'autre la réalité telle qu'elle apparaît à une conscience, telle qu'elle est pour nous, entre l'objet comme être en soi et l'objet comme objet d'une visée ou d'une perception, comme objet intentionnel. Il nous invite ainsi à ne plus identifier le vrai et l'objectif, tout en l'opposant au vécu et à l'apparent, et nous montre sans cesse comment le monde vécu est à l'origine du monde connu et à sa façon plus vrai que le vrai. On trouvera dans l'avantpropos de nombreux autres passages qui manifestent bien une telle orientation chez Merleau-Ponty.

Là où par exemple il définit la philosophie comme suit : « la philosophie n'est pas le reflet d'une vérité préalable, mais comme l'art la réalisation

63. M. Carbone, La visibilité de l'invisible: Merleau-Ponty, entre Cézanne et Proust, Hildesheim-Zürich-New York, G. Olms Verlag, 2001.

64. M. Merleau-Ponty, Phénoménologie de la perception, op. cit., p. 69. 
d'une vérité ${ }^{65}$. Au même titre que l'art moderne (par exemple la peinture de Cézanne), la philosophie ne se réfère pas à une image figée ou objective du monde en soi, ni n'est-elle l'expression vaine et gratuite d'une subjectivité : elle "veut comprendre comment paradoxalement il y a pour nous de l'en soi ${ }^{66}$, elle est, au même titre que l'art, une tentative risquée de « replacer sous notre regard » d'offrir «à notre constatation», le « rapport au monde, tel qu'il se prononce infatigablement en nous ${ }^{67}$, un effort pour traduire et exprimer dans un langage adéquat la texture originelle du monde et de notre vie, celle «qui paraît dans nos désirs, nos évaluations, notre paysage, plus clairement que dans la connaissance objective ${ }^{68}$, en tant que nous sommes au monde par notre corps.

Mais ce ne sont pas seulement les réflexions explicitement consacrées à la phénoménologie qui témoignent d'une étrange symbiose proposée par Merleau-Ponty, entre philosophie et art, d'une idée de la philosophie, assez proche de l'art et de la littérature. De fait, la Phénoménologie de la perception en elle-même, au travers de la parole, et de l'écriture, les procédés et les stratégies philosophiques qu'elle met en œuvre, témoigne d'une telle orientation et nous propose un exemple éclairant de cette proximité et d'une contamination féconde entre art et philosophie. On peut observer d'abord que Merleau-Ponty pratique ici une conceptualité descriptive et inachevable qui ne se tient ni aux critères cartésiens de l'évidence, ni à ceux, phénoménologiques, de l'universalité, mais au seul précepte husserlien de décrire le monde réel dans toutes ses formations, de "revenir "aux choses mêmes" ». On observe aussi que Merleau-Ponty utilise, pour s'exprimer, aussi bien des concepts thématiques, médités, techniques que ce que E. Fink appelait des concepts opératoires ${ }^{69}$, non réfléchis, couverts d'ombre (qui demeurent dans l'ombre), des notions transversales, des concepts qui, prima facie, ne conviennent pas, qui sont finalement étrangers au contexte et au vocabulaire philosophique, mais nous donnent en échange des représentations analogues, bref des images. Il apparaît en effet, dans la Phénoménologie, que, très souvent, l'interprétation d'une dimension de l'existence et de l'expérience emploie et utilise les catégories d'autres dimensions. L'on remarquera, également, qu'au cours d'un développement thématique, Merleau-Ponty guide souvent la compréhension du lecteur à travers d'innombrables exemples concrets ou détails, susceptibles de donner une véritable perception. L'on pourrait dire, finalement, que l'originalité, la force éclairante, la grandeur et le caractère impressionnant de cet ouvrage, qui nous donne le sentiment aigu de la réalité, résident en ceci que Merleau-Ponty cherche moins à créer ou à

65. Ibid., p. XV.

66. Ibid., p. 86.

67. Ibid., p. XIII.

68. Ibid.

69. E. Fink, «Les concepts opératoires dans la phénoménologie de Husserl », in Husserl. Cahiers de Royaumont, Philosophie, n. III, Paris, Éditions de Minuit, 1959, p. 214-241. 
exprimer des idées, qu'à "réveiller les expériences qui l'enracineront dans les autres consciences ${ }^{70}$. C'est pourquoi, à mon sens, l'on doit reconnaître qu'il s'agit d'un texte qui manifeste une conception de la philosophie aux ambitions strictement descriptives.

Il est temps de conclure. Il s'agit pour nous d'expliciter vers quelle nouvelle conception de la philosophie Merleau-Ponty nous amène au moins en intention. De notre discussion émerge clairement combien cet auteur s'éloigne des philosophes qui, depuis Platon, proclament d'une manière ou d'une autre le nécessaire dépassement du perçu, que ce soit au nom des Idées platoniciennes ou cartésiennes ou au nom des prétendus faits objectifs définis par la méthode de la science. Cette orientation ne semble toutefois pas nous entraîner en dehors de l'entreprise épistémologique au sens le plus large et inclusif, elle n'implique nullement une retombée du philosophique dans le métaphorique ou le poétique. Tout au contraire, comme en témoigne l'attention particulière qui lui est accordée par des philosophes qui réclament aujourd'hui un nécessaire dépassement du point de vue et de l'entreprise épistémologiques ${ }^{71}$, ce qui apparaît décisif et original dans le chemin indiqué et emprunté par Merleau-Ponty, c'est qu'il nous propose une façon d'assumer le projet moderne, tout en renversant le point de vue de l'épistémologie moderne classique.

70. M. Merleau-Ponty, Sens et non-sens, op. cit., p. 25.

71. Cf. à ce sujet, C. Taylor, Le dépassement de l'épistémologie, in J. Poulain, Critique de la raison phénoménologique, Paris, Les éditions du CERF, 1991, p. 115. 


\section{Bibliographie}

Barbaras R., Le tournant de l'expérience, Paris, Vrin, 1998.

Carbone M., La visibilité de l'invisible: Merleau-Ponty entre Cézanne et Proust, Hildesheim-Zürich-New York, G. Olms Verlag, 2001.

Fink E., «Les concepts opératoires dans la phénoménologie de Husserl », in Husserl. Cahiers de Royaumont, Philosophie, n. III, Paris, Éditions de Minuit, 1959.

Granel G., Traditionis traditio, Paris, Gallimard, 1972.

Merleau-Ponty M., Phénoménologie de la perception, Paris, Gallimard, 1945.

Merleau-Ponty M., Le primat de la perception et ses conséquences philosophiques, Lagrasse, Verdier, 1996.

Merleau-Ponty M., Sens et non-sens (1948) nouvelle édition, Paris, Gallimard, 1995.

Merleau-Ponty M., L'œil et l'esprit, Paris, Gallimard, 1964.

Merleau-Ponty M., Le visible et l'invisible, Paris, Gallimard, 1964.

Merleau-Ponty M., Parcours (1935-1951), Lagrasse, Verdier, 1997.

Merleau-Ponty M., Parcours II (1951-1961), Lagrasse, Verdier, 2000.

Taylor C., Le dépassement de l'épistémologie, in J. Poulain, Critique de la raison phénoménologique, Paris, Les éditions du CERF, 1991. 